\title{
Unintentional non-adherence to phosphate binders
}

\author{
Priya Patel, ${ }^{1}$ Sotiris Antoniou, ${ }^{2}$ Reena Popat ${ }^{3}$
}

- Additional material is published online. To view please visit the journal (http:// dx.doi.org/10.1136/ejhpharm2014-000456)

${ }^{1}$ Pharmacy Department, Barts Health NHS Trust, London, UK ${ }^{2}$ Cardiac Pharmacy Department, Barts Health NHS Trust, London, UK ${ }^{3}$ Sanofi, London, UK

\section{Correspondence to} Priya Patel, Pharmacy Department, Barts Health NHS Trust, Whitechapel Road, London E1 1BB, UK; pripatel@hotmail.co.uk

Received 19 February 2014 Accepted 30 May 2014 Published Online First 26 June 2014
CrossMark

To cite: Patel $P$ Antoniou S, Popat R. Eur J Hosp Pharm 2015;22: $18-22$.

\begin{abstract}
Objectives The aim of this study was to determine patient specific perceptual and practical barriers to adherence to phosphate binders. The second stage aimed to determine the effectiveness of an adherence enhancing strategy trialled by patients.
\end{abstract}

Methods A prospective, cross sectional study of patients attending a dialysis unit at a specialist centre, in central London, over a 6-month period (April-September 2013). Through patient questionnaires, an initial assessment was performed of specific barriers to adherence for each individual. Following this, an unintentional barrier to adherence was targeted: transportation of medication when away from home. Small pill pots were trialled by patients as an adherence enhancing strategy.

Results $13 \%$ patients admitted to not being adherent to their phosphate binders on a daily basis. The most common reasons reported were forgetting and difficulty in transportation of the medications when away from home. $94 \%$ patients claimed benefits to their adherence to phosphate binders as a result of the pill pot intervention.

Conclusions Non-adherence to phosphate binders continues to be an issue. With $94 \%$ patients claiming improved adherence with a smaller transportable pill pot, a potential patient-centred intervention has been explored to overcome an unintentional barrier.

\section{INTRODUCTION}

Medication adherence refers to whether patients take their medications as prescribed and continue to do so for the desired duration. Reports suggest that between $30 \%$ and $50 \%$ of all medications prescribed for long-term conditions such as chronic renal failure are not taken as recommended. ${ }^{1}$ This behaviour can adversely impact the course of the disease, as well as being an economic burden. The Department of Health (2010) estimates that wasted medicine costs the National Health Service (NHS) $£ 150$ million each year with poor adherence being a significant contributor. ${ }^{2}$ Furthermore, consequences of non-adherence to medications may result in adverse effects and lead to hospital admissions. It is estimated that $3 \%-4 \%$ of UK hospital admissions are as a result of avoidable medicine-related illness, with $11 \%-30 \%$ of these resulting from patients not adhering to their medications. ${ }^{3}$

Adherence is defined as 'the extent to which the patient's behaviour matches agreed recommendations from the prescriber'. ${ }^{4}$ Patients must believe there is a benefit to the prescribed medication and agree to take it as recommended; therefore, provider-patient communication is vital. Early studies have failed to define the characteristics that predict a 'non-adherent' patient. Instead, applying theoretical frameworks such as the perceptions and practicalities approach (PAPA) may allow us to identify patient specific barriers to adherence and tailor the response.

Chronic kidney disease patients have a high pill burden and non-adherence to medications is well documented and an ongoing issue..$^{5}$ In particular, phosphate binder medications have a reported low adherence rate with a systematic review of 34 studies reporting that 22\%-74\% (mean 51\%) patients are non-adherent to this group of medications. ${ }^{6}$

Crucially, the use of phosphate binders are associated with longer patient survival as they allow for less severe dietary protein restriction and improved nutritional status. ${ }^{7}$ Potential consequences of nonadherence to phosphate binders include hyperphosphataemia and the associated cardiovascular disease and bone disorders, ${ }^{8}$ for example, fractures and osteoporosis. Furthermore, the rare and often fatal condition calciphylaxis may be prevented through effective calcium phosphate control. ${ }^{9}$ A significantly higher parathyroid hormone (PTH) level has been observed for patient's non-adherent to phosphate binders in a recent study which was associated with increased cardiovascular mortality. ${ }^{7}$

Reasons for non-adherence to medications fall into two general overlapping categories: intentional and unintentional. Intentional non-adherence, when the patient decides not to take the medicine as recommended, is associated with patient's own beliefs and appraisals of the medicine and medicinetaking. Unintentional non-adherence is defined as occurring 'when a patient wants to take their medicine but is prevented from doing so by barriers beyond their control' (NICE guideline ${ }^{1}$ ). Horne ${ }^{10}$ identifies potential barriers to adherence as perceptual or practical. Reasons postulated for nonadherence to phosphate binders include side effects (most commonly gastrointestinal), which can interfere with social habits and lifestyle, and an increased pill burden. In a recent study exploring potentially modifiable factors associated with non-adherence to phosphate binders, a strong association between good patient-staff interaction and lower odds of non-adherence was recorded. ${ }^{7}$ Non-adherence may in part be a result of poor communication between the patient and the healthcare provider. ${ }^{11}$ In the 2009 medicines adherence guideline by the National Collaborating Centre for Primary Care, reasons for non-adherent behaviour included poor communication about health problems and potential treatments, and the patients' ongoing assessment and experience of treatments. ${ }^{4}$

Acknowledging that non-adherence to phosphate binders is an ongoing problem, a two stage audit to assess and attempt to improve adherence to phosphate binders in dialysis patients was conducted at 
Barts Health NHS Trust which cares for nearly 1000 haemodialysis patients.

\section{METHOD}

This is a prospective, cross sectional study of patients attending a dialysis unit at a specialist centre in central London, over a 6-month period (April-September 2013).

\section{STUDY POPULATION}

Information about patients who met the inclusion criteria was extracted from an internal database (Renalware, Filemaker). This central database for all renal patients under the care of Barts Health consists of patient demographics, relevant medical history and blood test results.

Inclusion criteria were patients undergoing haemodialysis on the dialysis unit prescribed one or more phosphate binders. Exclusion criteria included any patients with whom communication was a barrier (an interpreter was not used in this study), and patients who dialysed at home or on satellite units in the trust. For the second stage of the study, any patient who had been transplanted since the first stage was excluded alongside patients who had their phosphate binders dispensed in a medication dosage system or had been admitted as an inpatient during this section of the study period (September 2013).

\section{STUDY DESIGN}

A list of all haemodialysis patients was obtained from Renalware. An independent researcher took each of the recruited patients through the questionnaire and recorded their responses.

The questionnaire consisted of three sections and was based on the PAPA framework focusing attention on patient-centred interventions. ${ }^{10}$ An initial assessment was performed of specific perceptual and practical barriers for each individual. Patients were asked to rate their satisfaction with the amount of information they have received specific to their phosphate binders by a healthcare professional (see figure 1). This information was obtained using a validated tool: the Satisfaction with Information about Medicines Scale (SIMS). This is a 17-item questionnaire with potential responses ranging from none received to too much with an option to indicate that no information was required. Nine of these items relate to the actions and usage of medicines while eight relate to potential problems patients may experience. Four questions were not applicable with regard to phosphate binder counselling and were therefore removed from the questionnaire (regarding drowsiness, alcohol and sex life).

The second section of the questionnaire focused on how patients use their phosphate binders. This consisted of asking patients whether they were adherent on a daily basis and if so their understanding of the correct time to take their phosphate binders relative to their meals, that is, before, with or after meals. Lack of adherence identified at this stage was then investigated by asking the patient to select the reason(s) for nonadherence. Possible options included forgetting (unintentional non-adherence), altering the dose, stopping the tablets for a short period, missing out doses and taking less than instructed (intentional non-adherence). With all of these options, the auditor would be prompted to ask a set probing question in order to get a better understanding of the cause for nonadherence. Patients were also asked closed ended questions as to whether they found their phosphate binders too difficult to chew/swallow, unpleasant to taste, too difficult to transport, were not working or having bad side effects. A subsection on how patients manage their phosphate binder medication when going out was then completed with the final question as to whether they felt they would benefit from having a small pill pot for their phosphate binder medicine when they go out, that is, for meals or dialysis.

Demographic data were collected in the questionnaire and then supplemented with information from Renalware. At this point, phosphate levels were also collected for the recruited patients as a baseline (from Renalware). The initial work was evaluated to concentrate on one particular barrier to adherence and improve it. Recognition and development of adherence enhancing strategies can help people avoid error and improve performance.

Following our questionnaire reviewing patients' views, small pill pots were distributed to patients to assess whether this intervention would improve adherence when away from home. Between 20 and 30 tablets of the most commonly identified phosphate binders used in our patient population could be decanted into these pots. Patients were asked to trial the pill pot for 1 week and were then questioned on the benefits of the intervention if any. For patients on more than one phosphate binder, it was preagreed as to which one the pill pot should be used for.
Figure 1 Example questions from the questionnaire based on Satisfaction with Information about Medicines Scale (see online supplementary appendix 1 for full questionnaire).

\begin{tabular}{|c|c|c|c|c|c|}
\hline \multicolumn{6}{|c|}{$\begin{array}{l}\text { Please rate the information you have received about each of the following aspects of } \\
\text { your phosphate binder medicines. } \\
\text { 'None received' means you DID NOT RECEIVE any information and you would have liked } \\
\text { some information. } \\
\text { 'None needed' means you DID NOT NEED any information because you already knew } \\
\text { this or this was not important to you. }\end{array}$} \\
\hline & $\begin{array}{l}\text { Too } \\
\text { much }\end{array}$ & $\begin{array}{c}\text { About } \\
\text { right }\end{array}$ & $\begin{array}{l}\text { Too } \\
\text { little }\end{array}$ & $\begin{array}{l}\text { None } \\
\text { received }\end{array}$ & $\begin{array}{c}\text { None } \\
\text { needed }\end{array}$ \\
\hline $\begin{array}{l}\text { 1. What your phosphate binder } \\
\text { medicine is for }\end{array}$ & $\square$ & $\square$ & $\square$ & $\square$ & $\square$ \\
\hline 2. What it does & $\square$ & $\square$ & $\square$ & $\square$ & $\square$ \\
\hline 3. How it works & $\square$ & $\square$ & $\square$ & $\square$ & $\square$ \\
\hline 4. How long it will take to act & $\square$ & $\square$ & $\square$ & $\square$ & $\square$ \\
\hline 5. How you can tell if it is working & $\square$ & $\square$ & $\square$ & $\square$ & $\square$ \\
\hline
\end{tabular}




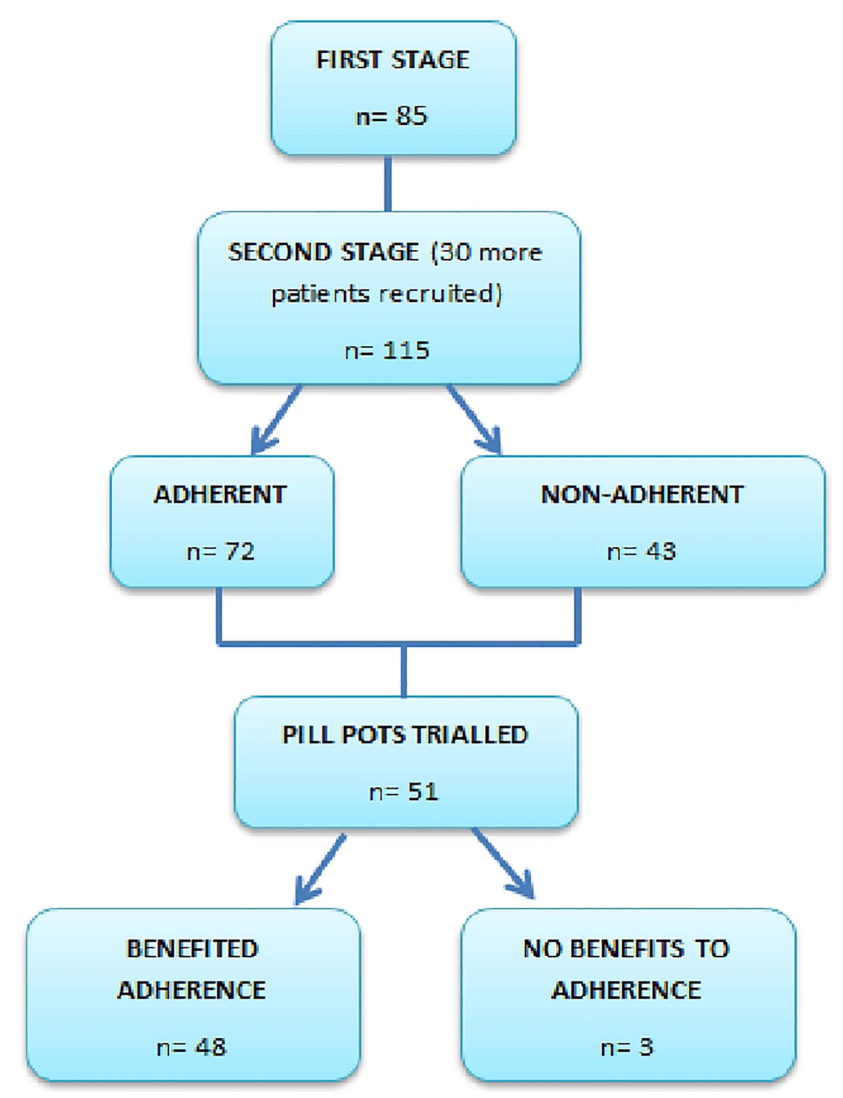

Figure 2 Flow diagram of the study design.

Following the 1-week trial, a questionnaire was used to gather responses with patients asked if they would prefer to complete the form themselves or be taken through it by the independent researcher. Patients were specifically asked if they had been adherent to their phosphate binder in the week they trialled the pill pot. If non-adherent, similar to the first stage questionnaire, patients were asked to select an option from forgetting, altering the dose, stopping the tablets for a short period, missing out doses and taking less than instructed. Patients were then asked if any of the options selected for their non-adherent behaviour was connected to the inability to transport the medication when away from home. The second part of the questionnaire asked the patients to compare their adherence before and after having the pill pot and whether they felt they had benefited from the intervention. Using Renalware, phosphate levels were collected for recruited patients in order to evaluate the impact of the intervention on laboratory parameters. Figure 2 summarises the study design.

This study was approved by the institution's research and development board.

\section{RESULTS}

A total of 85 patients were included in the first stage of the audit and taken through the questionnaire. A further 30 patients were recruited at the second stage of the audit in order to increase the sample size. All patients were questioned by the same independent researcher; however, only the first set recruited were asked the SIMS specific questions (section 1 of the questionnaire).

Figure 3 shows the responses of the 85 patients to the SIMS section. Patients felt they had 'Too much' or 'About right' amount of information with regard to how to use the phosphate binder medication and how to get a further supply.

Table 1 shows the characteristics of the patients by category of phosphate binder adherence. Compared with the adherent group of patients, the non-adherent group have a higher percentage of non-white male patients with a lower mean number of months on dialysis.

From the 115 patients in total recruited in the audit, $15(13 \%)$ admitted to being non-adherent to their phosphate binders on a daily basis. However, when specifically questioned

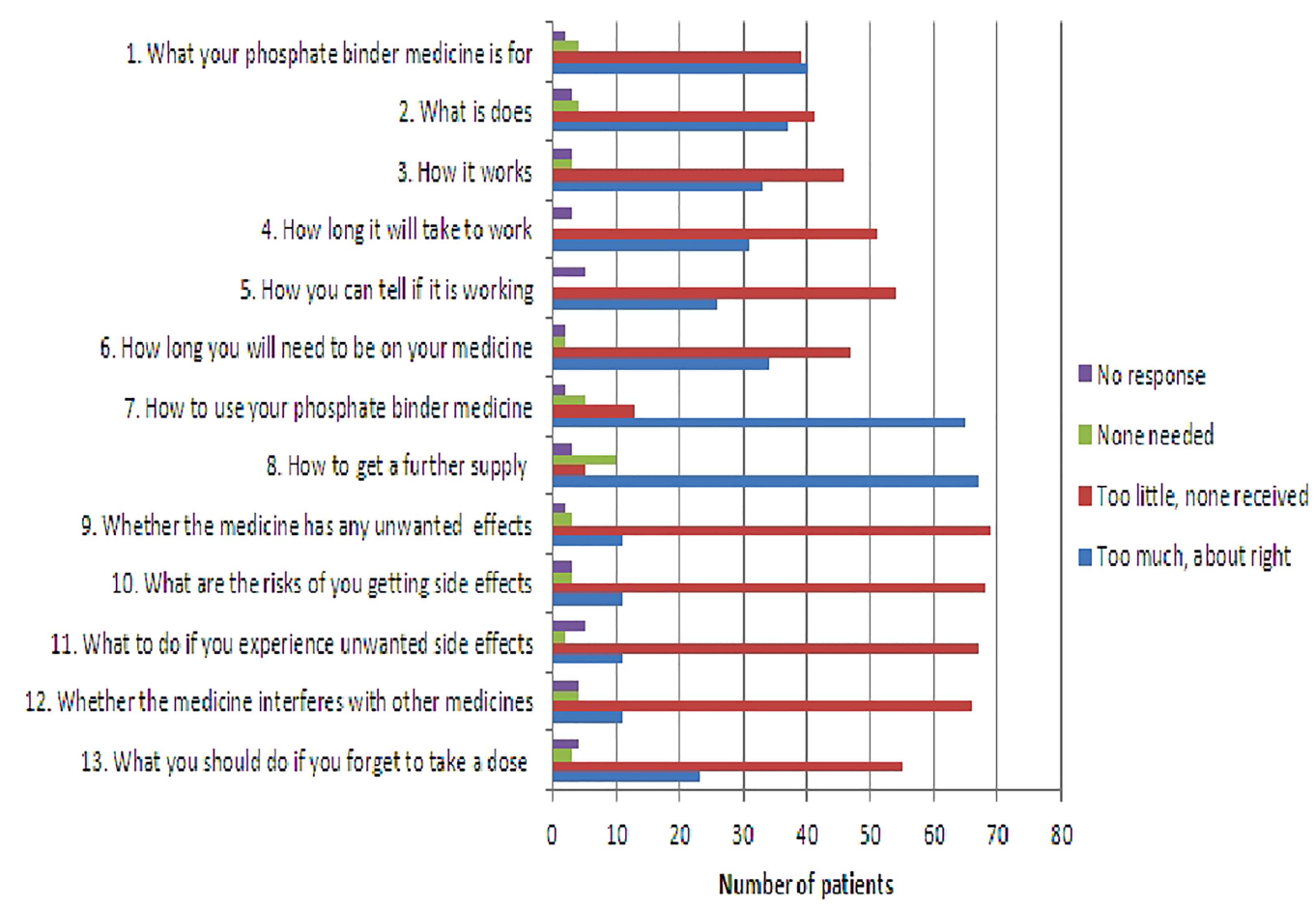

Figure 3 Responses from 85 patients to Satisfaction with Information about Medicines Scale questions. 
Table 1 Sociodemographic characteristics and comorbidities of the study patients $(n=115)$

\begin{tabular}{lll}
\hline Characteristics & Non-adherent $(\mathbf{n}=\mathbf{4 3})$ & Adherent $(\mathbf{n}=\mathbf{7 2})$ \\
\hline Age, years (mean) & 51.42 & 60.57 \\
\% Non-white patients & 79.1 & 54.2 \\
\% Male patients & 62.8 & 47.2 \\
Months on dialysis (mean) & 44.33 & 71.42 \\
\% Comorbidities & & \\
Diabetes & 30.2 & 30.6 \\
Cancer & 9.3 & 13.9 \\
Hypertension & 41.9 & 44.4 \\
Ischaemic heart disease & 14.0 & 20.8 \\
Heart failure & 7.0 & 0 \\
Peripheral vascular disease & 9.3 & 2.8 \\
Cerebrovascular accident & 7.0 & 4.2 \\
Depression (diagnosed) & 7.1 & 6.9 \\
\hline
\end{tabular}

on whether they took their phosphate binders when eating meals out of the house, 28 patients (24\%) said they did not. For the purposes of this audit, 43 patients $(37 \%)$ were considered to be non-adherent (self-reported non-adherence on a daily basis and/or non-adherence when eating meals out of the house). Patients who reported taking their phosphate binders on a daily basis and when away from home were considered to be adherent in this study. Reviewing phosphate levels, $47 \%$ of patients had levels outside of the desired range with $43 \%$ of these exceeding the upper limit.

The reasons for non-adherence reported are shown in figure 4. Forgetting to take the tablets was the major reason reported $(87 \%)$. With patients being counselled on the importance of taking these medications $5 \mathrm{~min}$ before or with their meals, many claimed to forget to do so at these very specific points in time and then omitting the dose. Figure 5 shows no link between the percentage of patients reporting non-adherence and an increasing pill burden.

In all, 32 patients $(28 \%)$ found the phosphate binder containers too difficult to transport when leaving the house. Those that did adhere would use methods such as wrapping tablets in tissue or cling-film or placing tablets loose in a pocket in order to transport them. Overall, $65 \%$ patients stated the provision of a small empty pill pot to decant the desired amount of phosphate binder tablets would be beneficial and improve their adherence.

In the second stage of the audit, a total of 68 pill pots were handed out to patients (one per patient). From the patients who met the inclusion criteria for the second stage of the study, some did not consent to trialling the pill pot as they were unwilling to do so or had a method of transporting their phosphate binder tablets which they were satisfied with. In all, 17 out of the 68 patients given pill pots did not use them and were therefore excluded from questioning on the impact to their adherence. Reasons for lack of use of the pill pots included forgetting to use it $(n=7)$ or not going out $(n=10)$. Overall, $94 \%(n=48)$ of all patients who trialled the pot for 1 week claimed that it benefited their ability to remain adherent to therapy. Comparison of 14 patients' phosphate levels from baseline blood tests and after 1 week of using the pill pot authenticated the findings that it increased adherence, with 64\% experiencing an appropriate reduction in their phosphate levels.

\section{DISCUSSION}

Complex treatment regimens along with silent benefits make phosphate binders a challenge to adhere to. Patients with a nonadherent tendency may choose to forgo this particular group of medications when they do not feel any obvious benefits. Similar to previous studies, this study showed that adherence to phosphate binders continues to be a challenge. Although the percentage of patients considered non-adherent in our audit was below the mean reported by Karamanidou et al, the methodology for assessing nonadherence differs and cannot be directly compared. ${ }^{6}$ Furthermore, if assessed against serum phosphate levels as in the case of many studies reviewed by Karamanidou et al, 47\% of patients in this study would be considered non-adherent with levels above the desired range $(0.8-1.6 \mathrm{mmol} / \mathrm{L})$. This would need further investigation as phosphate levels above the desired range may not be as a result of non-adherence but rather suboptimal therapy.

Identifying and targeting a practical, unintentional barrier to adherence in this audit through provision of small pill pots appears to have benefited the ease of transportation of phosphate binders for patients which is reflected in the appropriate reduction of phosphate levels. From patient feedback on the pill pots, the majority felt that a smaller, more compact container would be more suitable or a range of different size pots in order to suit individual lifestyles.

Figure 4 Reasons for non-adherence $(n=43)$.

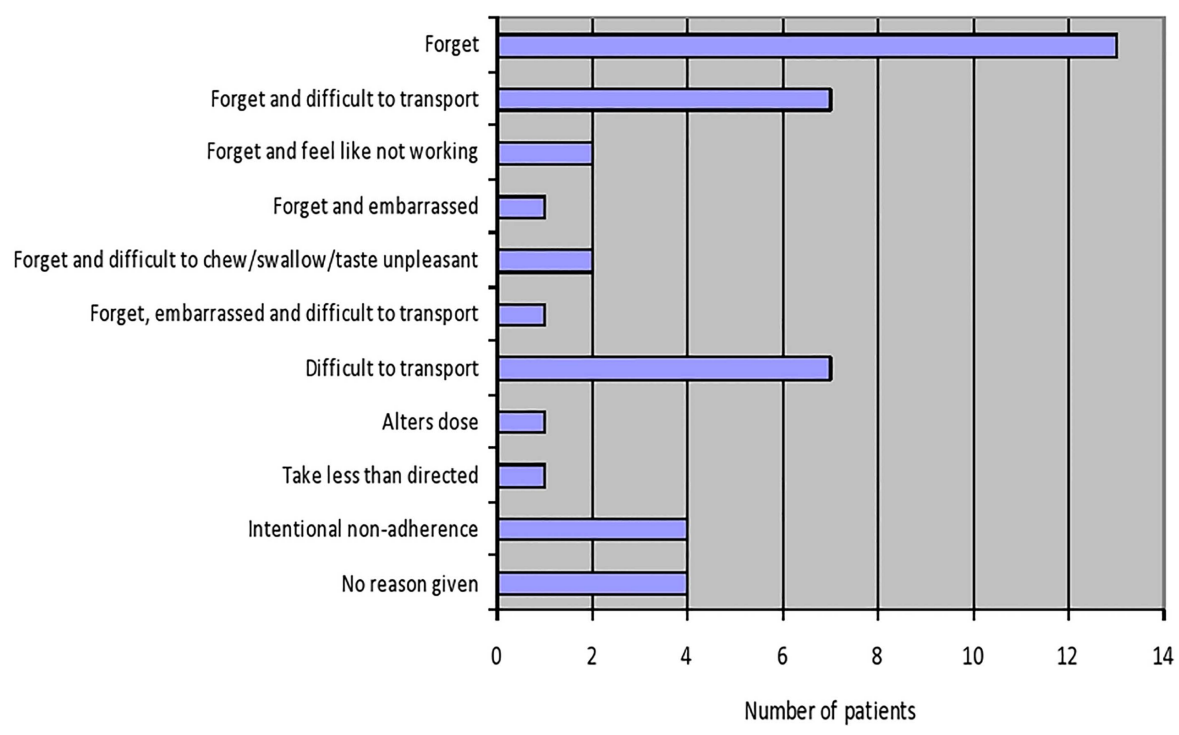




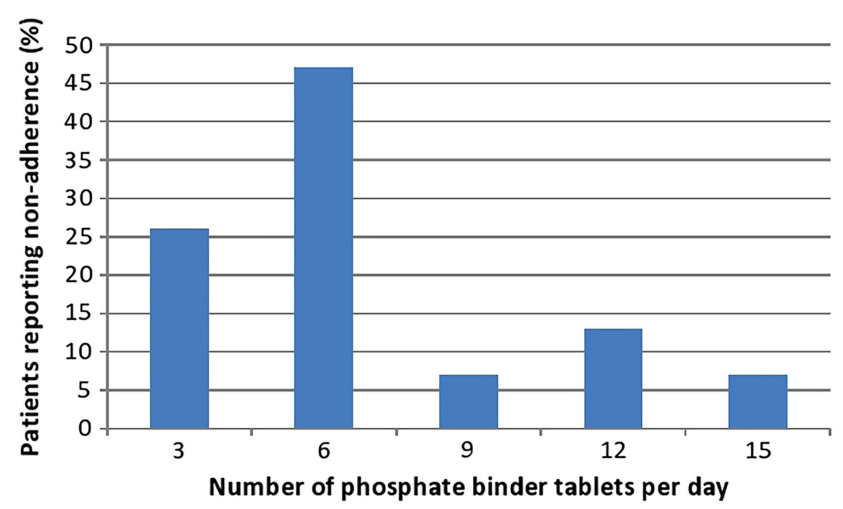

Figure 5 Prevalence of non-adherence by number of phosphate binder tablets.

Alongside easing transportation of tablets, many patients also reported having their phosphate binders in more than one container and location would act as a reminder. Leaving a pot of phosphate binders after use on the location where meals are generally consumed may remind patients to take the tablets at the next meal. Issues with this particular prompt include times when the patient eats meals away from their normal environment (eg, home) where the additional pill pot could provide an easy method of transportation of the tablets alongside giving the option of having the tablets in a handbag, the car or at the workplace (managing resource availability).

Creating cues to remember when to take phosphate binders is one example of a strategy to overcome forgetfulness; however, Furniss et $a l^{12}$ noted the quality of a cue depended on whether the cue is salient, linked to the topic to be recalled and task related. In the case of phosphate binders, cues such as a television programme or alarms may not be suitable unless patients consume their meals and snacks at the same time every day.

A lack of knowledge was identified throughout this audit with patients claiming inadequate counselling which contributed to their reduced satisfaction with information provision. This is of concern as lack of knowledge and understanding of health benefits from these medications could lead to non-adherence. If patients do not perceive phosphate binders to be an important group of medications, they are more likely to be non-adherent and less willing to attempt to overcome practical barriers such as forgetting or transportation issues. Likewise, resource barriers to adherence may reduce the motivation to take phosphate binder medications.

These findings suggest there is some work to be done surrounding patient education by healthcare professionals to improve patients' perceptions and subsequent adherence to phosphate binders. It is evident that patients do not feel they received sufficient counselling on most aspects of their phosphate binder medications. This is also supported by the difference in self-reporting of non-adherence when patients were asked if they took their phosphate binders on a daily basis and then asked if they took their binders when they were away from home. With an almost twofold increase in non-adherence when patients were away from home, this may imply that patients do not consider themselves as non-adherent when eating meals or snacks outside of their home perhaps due to a lack of knowledge of when they need to take their binders or self-belief that they are not crucial.

Limitations in this audit include the small sample size and lack of blood tests for all patients. Alongside this, there is an element of subjectivity with adherence based on self-reporting.
Many of the patients who agreed to trial the pill pot were also doing Ramadan and did not eat meals or snacks away from home and subsequently failed to use the pot during the trial week. The intervention would need to be trialled in a larger sample size with recording of a full bone profile for a better indication of its impact. Language barriers with many of the potential recruits meant they were excluded or may not have fully understood some of the questions.

\section{CONCLUSIONS}

With 48 patients (94\%) claiming improved adherence with a smaller transportable pill pot, a potential patient-centred intervention has been explored to overcome an unintentional barrier. Alongside this, integrating an educational based approach to medicine support is essential in overcoming non-adherence to this vital group of medications.

\section{Key messages}

Studies show that $22 \%-74 \%$ (mean $51 \%$ ) of patients are non-adherent to phosphate binder medications.

- The use of phosphate binders is associated with longer patient survival.

- Good patient-healthcare professional interaction has been shown to lower odds of non-adherence, but specific characteristics of a non-adherent patient have failed to be defined.

- The major reasons identified for non-adherence to phosphate binder medication in this study were forgetfulness $(87 \%)$ and difficulty with transportation of the medications particularly when away from home (28\%).

- Patient-centred interventions, for example, use of a small transportable pill pot, can overcome unintentional reasons for non-adherence.

Contributors PP: design, execution, interpretation of data and original draft of write up. SA: conception and design, review and editing of paper. RP: conception and design, review and editing of paper.

Ethics approval Barts Health NHS Trust.

Provenance and peer review Not commissioned; externally peer reviewed.

\section{REFERENCES}

1 National Institute for Health and Care Excellence. [Medicines Adherence. Involving patients in decisions about prescribed medicines and supporting adherence]. [CG76]. London: National Institute for Health and Care Excellence, 2009.

2 http://www.hsj.co.uk/resource-centre/best-practice/qipp-resources/ how-improved-medication-adherence-can-prevent-costly-medicine-waste/5041067. article. How improved medication adherence can prevent costly medicine waste.

3 http://www.nice.org.uk/nicemedia/pdf/CG76CostStatement.pdf

4 http://www.nice.org.uk/nicemedia/live/11766/42971/42971.pdf

5 http://www.ncbi.nlm.nih.gov/pmc/articles/PMC2935303/

6 Karamanidou C, Clatworthy J, Weinman J, et al. A systematic review of the prevalence and determinants of nonadherence to phosphate binding medication in patients with end-stage renal disease. BMC Nephrology 2008;9:2.

7 Martins MT, Silva LF, Kraychete A, et al. Potentially modifiable factors associated with non-adherence to phosphate binder use in patients on haemodialysis. BMC Nephrology 2013;14:208.

8 http://www.kidney.org/professionals/kdoqi/guidelines_ckd/p6_comp_g10.htm

9 http://ckj.oxfordjournals.org/content/3/1/68.full

10 Horne R. Advances in Asthma Management E-book Improving adherence with Asthma therapies. http://www.futuremedicine.com

11 http://www.nice.org.uk/nicemedia/pdf/cg76fullguideline.pdf

12 Furniss D, Barber N, Lyons I, et al. Unintentional non-adherence can a spoon full of resilience help the medicine go down? BMJ Qual Saf 2014;23:95-8. 